ESAIM: COCV 27 (2021) 39

https://doi.org/10.1051/cocv/2021037
ESAIM: Control, Optimisation and Calculus of Variations

www.esaim-cocv.org

\title{
A SINGULAR ELLIPTIC EQUATION AND A RELATED FUNCTIONAL*
}

\author{
A. Ferone ${ }^{1}$, A. Mercaldo ${ }^{2, * *}$ and S. Segura de León ${ }^{3}$
}

\begin{abstract}
We study a class of Dirichlet boundary value problems whose prototype is

$$
\begin{cases}-\Delta u=|u|^{p-2} u+f(x) & \text { in } \Omega, \\ u=0 & \text { on } \partial \Omega,\end{cases}
$$

where $0<p<1$ and $f$ belongs to a suitable Lebesgue space. The main features of this problem are the presence of a singular term $|u|^{p-2} u$ and a datum $f$ which possibly changes its sign. We introduce a notion of solution in this singular setting and we prove an existence result for such a solution. The motivation of our notion of solution to problem above is due to a minimization problem for a nondifferentiable functional on $H_{0}^{1}(\Omega)$ whose formal Euler-Lagrange equation is an equation of that type. For nonnegative solutions a uniqueness result is obtained.
\end{abstract}

Mathematics Subject Classification. 35B25, 35B27, 35J25, 35J67.

Received July 26, 2019. Accepted April 1, 2021.

\section{INTRODUCTION}

In the present paper we deal with a semilinear elliptic equation having a singularity. We look for a function $u$ which solves the problem

$$
\begin{cases}-\operatorname{div}(A(x) \nabla u)=g(x, u)+f(x), & \text { in } \Omega \\ u=0, & \text { on } \partial \Omega\end{cases}
$$

* The third author is partially supported by the Spanish Ministerio de Ciencia, Innovación y Universidades and FEDER, under project PGC2018-094775-B-I00. The first and second authors are partially supported by INdAM - GNAMPA

Keywords and phrases: Semilinear equations, singularity at $u=0$, existence, uniqueness.

${ }^{1}$ Dipartimento di Matematica e Fisica, Università degli Studi della Campania "L. Vanvitelli" Viale Lincoln 5, 81100 Caserta, Italy.

2 Dipartimento di Matematica e Applicazioni "R. Caccioppoli", Università degli Studi di Napoli Federico II, Complesso Monte S. Angelo, Via Cintia, 80126 Napoli, Italy.

3 Departament d'Anàlisi Matemàtica, Universitat de València, Dr. Moliner 50, 46100 Burjassot, València, Spain.

** Corresponding author: mercaldo@unina.it 
where $\Omega$ is an open bounded set of $\mathbb{R}^{N}, N \geq 2, A(x)$ is a symmetric matrix $A(x)=\left(a_{i j}(x)\right)$ such that $a_{i j} \in$ $L^{\infty}(\Omega)$ and verifies the ellipticity condition

$$
A(x) \xi \cdot \xi \geq \lambda|\xi|^{2}
$$

for all $\xi \in \mathbb{R}^{N}$, for almost every $x \in \Omega$ and for some $\lambda>0$. Moreover $g$ is a singular function at $s=0$, that is we assume that

$$
g: \Omega \times(\mathbb{R} \backslash\{0\}) \longrightarrow \mathbb{R}
$$

is a Carathéodory function which satisfies the following growth condition:

$$
|g(x, s)| \leq \Lambda|s|^{p-1},
$$

where $0<p<1$ and $\Lambda>0$, for almost all $x \in \Omega$ and for all $s \in \mathbb{R} \backslash\{0\}$.

Finally we assume that the datum $f$ can change its sign and satisfies the following summability assumption

$$
f \in L^{m}(\Omega), \quad m>\frac{N}{2} .
$$

The basic model for the function $g(x, s)$ is given by

$$
g(x, s)=\frac{h(x) s}{|s|^{2-p}}, \quad \text { for a.e. } x \in \Omega \text { and for all } s \in \mathbb{R} \backslash\{0\},
$$

with $h$ a function belonging to $L^{\infty}(\Omega)$ and $0<p<1$.

As far as existence results for this type of problems are concerned, we recall the classical paper [11], where the authors prove the existence of a classical positive solution if the matrix $A(x)$, the boundary $\partial \Omega$ and the function $g(x, s)+f(x)$ are smooth enough.

The study of different features of positive solutions to the Dirichlet problem for equations of type $-\Delta u=$ $u^{-\gamma}+f(x, u)$ is addressed in several papers (see [7-9]) from a variational point of view.

In [5] the authors consider the case where $f \equiv 0, g(x, s)=\frac{h(x)}{s^{\gamma}}$, with $\gamma>0$ and $h$ a nonnegative function belonging to a suitable Lebesgue space. They prove existence, uniqueness and regularity results for positive distributional solutions. Main tools in such an approach are the assumption that $g(x, s)$ is nonincreasing in the variable $s$ and the maximum principle. Uniqueness and comparison results for this type of solution has been proved in [4] and [10], this one with symmetrization techniques.

A different approach is used in the papers [15-17] where existence, uniqueness and stability results are proved without assuming that $g(x, s)$ is an nonincreasing function in the variable $s$ and without using the maximum principle in the proofs. In $[15,16]$ the case where $f \equiv 0$ and $g(x, s)=\frac{h(x)}{s^{\gamma}}$, with $0<\gamma \leq 1$ and $h$ belonging to a Lebesgue space, is studied, while the case $\gamma>1$ is considered in [17]. In the latest case, no global energy estimate is available for solutions and this leads to introduce a notion in the spirit of the notion of "solution defined by transposition" introduced in other problems by J.-L. Lions and E. Magenes, and by G. Stampacchia. Such a notion cannot be extended to the case of nonlinear operators, except for some special cases.

Further contributions to semilinear elliptic equations having this type of singularity are contained for example in $[1-3,8,9,12,21,22]$.

A common feature to all these papers is that only positive solutions are considered. A very few results are known about existence of solutions which changes its sign and a first paper in this direction is [6], where the authors show that if the "singular term" goes to infinity at zero faster than $1 /|u|$ then only nonnegative solutions are possible, while in the other case nonpositive solution or even solutions changing the sign are possible. 
In the present paper we are concerned with existence and uniqueness for the singular problems of the type (1.1). Our interest was inspired by the study of existence of solutions to problem (1.1) changing the sign. This induces us to study the minimization problem for the following functional on $H_{0}^{1}(\Omega)$

$$
\mathcal{J}[u]=\frac{1}{2} \int_{\Omega} A(x) \nabla u \cdot \nabla u-\int_{\Omega} \mathcal{G}(x, u)-\int_{\Omega} f(x) u
$$

where $\mathcal{G}(x, s)$ is a primitive of $g(x, s)$, that is

$$
\mathcal{G}(x, s)=\int_{0}^{s} g(x, t) \mathrm{d} t, \quad s \in \mathbb{R} .
$$

We prove that a solution to problem (1.1) is given by a minimizer of this functional defined on $H_{0}^{1}(\Omega)$. Observe that (1.1) is formally the Euler-Lagrange equation of this functional. Actually, this is true in the sense proved in Proposition 3.2 below.

A first difficulty arises when we deal with the singular term since we cannot prove it belongs to $L_{l o c}^{1}(\Omega)$. It implies that the distributional formulation of problem (1.1) is not available. This hindrance occurs even if $g(x, u) \in L_{l o c}^{1}(\Omega)$ and begs the question of identifying the right concept of solution to problem (1.1). Inspired by the minimization problem of (1.6), we introduce a concept based on a class of test functions whose main feature is that they vanish when $u$ vanishes. Our concept resembles that of "renormalized solution" introduced by P.-L. Lions and F. Murat in [20] (see also [13]) for nonlinear elliptic equation having $L^{1}$-function or measure data. In this formulation test functions are chosen as $S(u) \varphi$ to cut solutions at infinity and a condition must be added to control the energy as solutions go to infinity. This condition reads as

$$
\lim _{k \rightarrow+\infty} \frac{1}{k} \int_{\{k<|u|<2 k\}} A(x) \nabla u \cdot \nabla u \mathrm{~d} x=0
$$

when the measure datum is singular with respect to the capacity. We point out that, as a consequence of this condition, every renormalized solution is a distributional one.

In our setting, obviously, test functions must cut solutions at zero (where the singularity is located). Having identified our notion of solution, then an important question arises, namely, to show when a solution to problem (1.1) in our sense is actually a distributional solution. In the case $g(x, u) \in L_{l o c}^{1}(\Omega)$, the condition

$$
\lim _{k \rightarrow 0} \frac{1}{k} \int_{\{|u|<k\}} A(x) \nabla u \cdot \nabla u \mathrm{~d} x=0
$$

provides us of a sufficient condition to ensure what solutions of problem (1.1) (according to Def. 2.1) are distributional ones.

We have mentioned that test functions must cut solutions at zero, and this leads to another drawback. Indeed, the function that vanishes identically always satisfies our formulation and this fact raises the question of proving that the solution we find is not the trivial solution. We are able to check it when the singular term satisfies a sign condition, namely: $g(x, s) \cdot S \geq 0$ for every $s \in \mathbb{R}$.

On the other hand, our definition of solution is also based on the fact that our solution is bounded (see Def. 2.1). The necessity of dealing with bounded functions justifies the assumption that the data $f$ have to belong to Lebesgue spaces $L^{m}(\Omega)$, with $m>N / 2$. Nevertheless in a forthcoming paper we show that our definition of solution can also be adapted to the case of datum $f$ belonging to a larger class of Lebesgue spaces and to a class of nonlinear elliptic operators whose principal part is a general Leray-Lions type operator.

When dealing with positive solutions is possible to arrive at a uniqueness result assuming $g(x, s)$ is nonincreasing in the variable $s$. This fact is easy to check for distributional solutions. We will prove it for 
nonnegative solutions which satisfy a slightly milder condition than (1.8). Uniqueness of nonnegative solutions to problem (1.1) are also proven in Section 5. There, we consider the following problem

$$
\begin{cases}-\operatorname{div}(A(x) \nabla u)=g(x, u)+f(x), & \text { in } \Omega \\ u>0, & \text { in } \Omega \\ u=0, & \text { on } \partial \Omega,\end{cases}
$$

where we assume that $g(x, s)$ satisfies

$$
0 \leq g(x, s) \leq \Lambda s^{p-1}
$$

with $0<p<1$ and $\Lambda>0$, for almost all $x \in \Omega$ and for all $s \geq 0$.

This paper is organized as follows. In Section 2 we introduce our notion of solution and state our main results.

The minimization problem for the functional (1.6) is treated in Section 3 where we specify in which sense problem (1.1) is the Euler-Lagrange equation of functional (1.6) and prove the existence of a minimizer.

In Section 3 we also prove that $u$ is a solution to problem (1.1) in the sense of Definition 2.1 below.

In Section 4 we study the relation between distributional solutions and our definition of solution and we give a sufficient condition which makes the solution we have found a solution in the sense of distributions.

In Section 5 we face the study of nonnegative solutions to problem (1.1). We give a sufficient condition for the uniqueness of such a solution.

Finally in Section 6 we make some remarks concerning our approach and the standard approximation procedure, which seems not significant in this setting.

\section{Notation And STATEMEnts of REsults}

Throughout this paper, $\Omega$ stands for an open bounded set of $\mathbb{R}^{N}$, with $N \geq 1$. The Lebesgue measure of $E \subset \Omega$ will be denoted by $|E|$. The symbols $L^{q}(\Omega)$ will denote the usual Lebesgue spaces and $H_{0}^{1}(\Omega)$ the usual Sobolev space, of measurable functions having weak derivative in $L^{2}\left(\Omega ; \mathbb{R}^{N}\right)$ and zero trace on $\partial \Omega$.

On the other hand, the positive and negative part of a function $u$ is denoted by $u_{+}$and $u_{-}$, respectively. Moreover, we denote

$$
\{|u| \geq \delta\}=\{x \in \Omega:|u(x)| \geq \delta\}
$$

for any $\delta>0$.

In what follows, we will also consider two auxiliary functions. For any $s \in \mathbb{R}$ and any $k>0$ we define

$$
\begin{gathered}
G_{k}(s)=(|s|-k)_{+} \operatorname{sign}(s), \\
T_{k}(s)=\max \{-k, \min \{s, k\}\} .
\end{gathered}
$$

The aim of this section is to introduce our notion of solution and to state our main results.

\subsection{Definition of solution to problem (1.1)}

As pointed out in the Introduction, a notion of solution to problem (1.1) has to be defined. The following definition is based on a suitable class of functions test, whose role is to cut the zone where the solution $u$ is zero. We emphazise the analogy with the definition of renormalized solution introduced by P.L. Lions and F. Murat for nonlinear elliptic equations with $L^{1}$-data or measures data (see, for example, [13, 20]). 
Definition 2.1. We will say that $u \in H_{0}^{1}(\Omega) \cap L^{\infty}(\Omega)$ is a solution to (1.1) if

$$
\frac{|\nabla u|^{2}}{|u|^{p}} \in L^{1}(\Omega)
$$

and

$$
\int_{\Omega} S^{\prime}(u) \varphi A(x) \nabla u \cdot \nabla u+\int_{\Omega} S(u) A(x) \nabla u \cdot \nabla \varphi=\int_{\Omega} g(x, u) S(u) \varphi+\int_{\Omega} f S(u) \varphi .
$$

for every Lipschitz-continuous $S: \mathbb{R} \rightarrow \mathbb{R}$ satisfying $S(0)=0$ and for every $\varphi \in H^{1}(\Omega) \cap L^{\infty}(\Omega)$. Here we have defined $g(x, 0)$ in such a way that $g(x, 0) S(0)=0$.

Remark 2.2. It is worth remarking that no singularity occurs in the product $|g(x, s)||s|^{1-p}$ since by (1.3),

$$
|g(x, s)||s|^{1-p} \leq \Lambda, \quad s \neq 0 .
$$

Obviously the product $g(x, s) s$ has also no singularities. More generally the product $g(x, s) S(s)$ has no singularities and therefore we can define the function $g(x, \cdot)$ in 0 in such a way that $g(x, 0) S(0)=0$.

Remark 2.3. Observe that, since $S$ is a Lipschitz continuous function such that $S(0)=0$ and $\varphi \in H^{1}(\Omega) \cap$ $L^{\infty}(\Omega)$, we get $S(u) \varphi \in H_{0}^{1}(\Omega)$. This implies that the first two terms in the left-hand side of (2.4) are welldefined. Moreover, by condition $|S(u)| \leq L|u|$, the growth condition on $g(1.3)$ and since $g(x, 0) S(0)=0$, we get

$$
\left|\int_{\Omega} g(x, u) S(u) \varphi \mathrm{d} x\right| \leq L\|\varphi\|_{L^{\infty}} \int_{\Omega}|u|^{p} \mathrm{~d} x<+\infty .
$$

Finally since $S(u) \varphi \in L^{\infty}(\Omega)$ also the last term on the right-hand side is finite.

Moreover we explicitly remark that, as a consequence of (2.3), the formulation (2.4) holds for every function $S: \mathbb{R} \rightarrow \mathbb{R}$ which is Lipschitz-continuous on every closed interval which does not contain 0 , satisfying $S(0)=0$ and $|S(s)| \leq L|s|^{1-p}$ for certain $L>0$.

Remark 2.4. We point out that $u \equiv 0$ always satisfies requirement (2.4). In (3.8), we introduce a condition which avoids the possibility of considering the trivial solution.

Remark 2.5. We remark that, in general, the singular term $g(x, u)$ in the problem (1.1) is not a summable function, i.e.

$$
g(x, u) \notin L^{1}(\Omega) .
$$

Notice, however, that any solution $u$ to problem (1.1) in the sense of Definition 2.1 satisfies

$$
g(x, u) \chi_{\{|u| \geq \delta\}} \in L^{1}(\Omega) \quad \forall \delta>0 .
$$

Indeed this is an easy consequence of the growth condition on $g(x, s)(1.3)$ and the fact that $p<1$.

\subsection{Statements of main results}

We are interested in the existence of changing sign solutions to problem (1.1) jointly with the minimization of the functional (1.6).

Our main results are stated as follows. 
Theorem 2.6. Under the assumptions (1.2), (1.3) and (1.4), problem (1.1) has at least a solution in the sense of Definition 2.1 which minimizes functional (1.6).

The second main result concerns uniqueness of nonnegative solutions:

Theorem 2.7. Assume (1.2) and (1.10). If

$$
g(x, \cdot) \text { is a nonincreasing function in } s \in \mathbb{R}_{+}
$$

for a.e. $x \in \Omega$ and $u_{1}, u_{2}$ are nonnegative solutions to problem (1.9) in the sense of Definition 2.1 which satisfy the condition

$$
\lim _{\delta \rightarrow 0} \frac{1}{\delta} \int_{\left\{\delta \leq u_{i} \leq 2 \delta\right\}} A(x) \nabla u_{i} \cdot \nabla u_{i} \mathrm{~d} x=0, \quad i=1,2
$$

then $u_{1}=u_{2}$ a.e. in $\Omega$.

Remark 2.8. We stress that our results also hold for more general functions. For instance, we may replace condition (1.3) with

$$
|g(x, s)| \leq \Lambda_{1}|s|^{p-1}+\Lambda_{2} .
$$

In particular, it is not necessary that $\lim _{|s| \rightarrow \infty} g(x, s)=0$.

\section{EXISTENCE RESUlT}

This section is devoted to obtain a minimizer of functional $\mathcal{J}$ which is also a solution to problem (2.6). To this end, we firstly prove that such a minimizer exists and then we derive the Euler-Lagrange equation of this functional which is equation in (2.6), as expected.

Proposition 3.1. Under the assumptions (1.2), (1.3) and (1.4), there exists $u \in H_{0}^{1}(\Omega) \cap L^{\infty}(\Omega)$ that minimizes functional $\mathcal{J}$, defined in (1.6).

Proof. Observe that Young's inequality implies

$$
\begin{aligned}
\frac{1}{2} A(x) \nabla u \cdot \nabla u-\mathcal{G}(x, u)-f(x) u \geq \frac{\lambda}{2}|\nabla u|^{2}-\frac{\Lambda}{p}|u|^{p}-|f(x)||u| & \\
& \geq \frac{\lambda}{2}|\nabla u|^{2}-\left[\frac{\Lambda}{2}+\frac{1}{2}|f|\right]|u|^{2}-\left[\frac{\Lambda(2-p)}{2 p}+\frac{1}{2}|f|\right]
\end{aligned}
$$

where the functions into brackets belong to $L^{m}(\Omega)\left(m>\frac{N}{2}\right)$. A similar inequality majorizing the integrand of $\mathcal{J}$ holds. Now, our result is a straightforward consequence of well-known results of the Calculus of Variations (see, for instance, [19]).

Proposition 3.2. Assume that $u \in H_{0}^{1}(\Omega) \cap L^{\infty}(\Omega)$ is a minimizer of the functional $\mathcal{J}$ defined in (1.6). Then $u$ satisfies the identity

$$
\int_{\Omega} S^{\prime}(u) \varphi A(x) \nabla u \cdot \nabla u \mathrm{~d} x+\int_{\Omega} S(u) A(x) \nabla u \cdot \nabla \varphi \mathrm{d} x=\int_{\Omega} g(x, u) S(u) \varphi \mathrm{d} x+\int_{\Omega} f S(u) \varphi \mathrm{d} x,
$$

for any $S: \mathbb{R} \rightarrow \mathbb{R}$ Lipschitz-continuous function such that $S(0)=0$ and for any $\varphi \in H^{1}(\Omega) \cap L^{\infty}(\Omega)$. (Recall that we have defined $g(x, 0)$ in such a way that $g(x, 0) S(0)=0$.) 
Proof. Consider a Lipschitz-continuous function $S: \mathbb{R} \rightarrow \mathbb{R}$ such that $S(0)=0$ and fix $\varphi \in H^{1}(\Omega) \cap L^{\infty}(\Omega)$. The following equality holds

$$
\begin{aligned}
\frac{\mathcal{J}[u+t S(u) \varphi]-\mathcal{J}[u]}{t} & =\frac{1}{2 t} \int_{\Omega}(A(x) \nabla(u+t S(u) \varphi) \cdot \nabla(u+t S(u) \varphi)-A(x) \nabla u \cdot \nabla u) \\
& -\frac{1}{t} \int_{\Omega}(\mathcal{G}(x, u+t S(u) \varphi)-\mathcal{G}(x, u))-\int_{\Omega} f S(u) \varphi=\mathcal{J}_{1}(t)-\mathcal{J}_{2}(t)-\int_{\Omega} f S(u) \varphi,
\end{aligned}
$$

where $\mathcal{G}(x, \cdot)$ denotes the primitive of $g(x, \cdot)$ as in $(1.7)$,

$$
\begin{gathered}
\mathcal{J}_{1}(t)=\frac{1}{2 t} \int_{\Omega}(A(x) \nabla(u+t S(u) \varphi) \cdot \nabla(u+t S(u) \varphi)-A(x) \nabla u \cdot \nabla u), \\
\mathcal{J}_{2}(t)=\frac{1}{t} \int_{\Omega}(\mathcal{G}(x, u+t S(u) \varphi)-\mathcal{G}(x, u)) .
\end{gathered}
$$

Let us evaluate $\mathcal{J}_{1}(t)$ and $\mathcal{J}_{2}(t)$. The case $\mathcal{J}_{1}(t)$ follows by a classical argument (see, for instance, [14], Chap. 8): It is easy to verify that

$$
\mathcal{J}_{1}(t)=\int_{\Omega} S^{\prime}(u) \varphi A(x) \nabla u \cdot \nabla u+\int_{\Omega} S(u) A(x) \nabla u \cdot \nabla \varphi+\frac{t}{2} \int_{\Omega} A(x) \nabla(S(u) \varphi) \cdot \nabla(S(u) \varphi) .
$$

We explicitly remark that all terms are well-defined since $S$ is Lipschitz-continuous and $u$ is bounded.

Therefore letting $t \rightarrow 0$, we obtain

$$
\lim _{t \rightarrow 0} \mathcal{J}_{1}(t)=\int_{\Omega} S^{\prime}(u) \varphi A(x) \nabla u \cdot \nabla u+\int_{\Omega} S(u) A(x) \nabla u \cdot \nabla \varphi
$$

Now let us evaluate $\mathcal{J}_{2}(t)$, the second term in (3.2). We claim that

$$
\lim _{t \rightarrow 0} \mathcal{J}_{2}(t)=\int_{\Omega} g(x, u) S(u) \varphi \mathrm{d} x
$$

It is obvious that

$$
\lim _{t \rightarrow 0} \frac{\mathcal{G}(x, u+t S(u) \varphi)-\mathcal{G}(x, u)}{t}=g(x, u) S(u) \varphi, \quad \text { a.e. in } \Omega
$$

Then, in order to apply Lebesgue's dominated convergence Theorem, we will prove that, for $t$ small enough,

$$
\left|\frac{\mathcal{G}(x, u+t S(u) \varphi)-\mathcal{G}(x, u)}{t}\right| \leq 2^{1-p} \Lambda L\|u\|_{\infty}^{p}\|\varphi\|_{\infty}
$$

where $L>0$ is a constant satisfying $|S(u)| \leq L|u|$. To this aim, we write

$$
\frac{\mathcal{G}(x, u+t S(u) \varphi)-\mathcal{G}(x, u)}{t}=\frac{1}{t}\left(\int_{0}^{t} g(x, u+\tau S(u) \varphi) S(u) \varphi d \tau\right) .
$$


Now choose $t$ satisfying

$$
L|t|\|\varphi\|_{\infty} \leq \frac{1}{2}
$$

By assumption (1.3), we get

$$
\begin{aligned}
& |g(x, u+\tau S(u) \varphi) S(u) \varphi| \leq\left|\frac{\Lambda}{|u+\tau S(u) \varphi|^{1-p}} S(u) \varphi\right| \\
& \quad \leq \frac{\Lambda L|u|\|\varphi\|_{\infty}}{(|u|-L|\tau||u||\varphi|)^{1-p}} \\
& \quad \leq \frac{L|u|^{p}}{\left(1-L|t|\|\varphi\|_{\infty}\right)^{1-p}} \Lambda\|\varphi\|_{\infty} \leq 2^{1-p} L\|u\|_{\infty}^{p} \Lambda\|\varphi\|_{\infty} .
\end{aligned}
$$

By (3.6) this yields (3.5) and therefore, via Lebesgue's dominated convergence theorem, the assertion (3.4). Collecting (3.2),(3.3) and (3.4) we deduce the conclusion.

Proof of Theorem 2.6. Only assertion (2.3) must be checked. To this end, for any fixed $k>0$ we consider $S(u)=G_{k}\left(|u|^{-p} u\right)$ and $\varphi=1$. Then, by ellipticity condition $(1.2)$, since $g(x, 0) G_{k}(0)=0$, we get

$$
\begin{aligned}
(1-p) \lambda & \int_{\left\{|u|^{1-p}>k\right\}}|u|^{-p}|\nabla u|^{2} \mathrm{~d} x \\
& \leq \int_{\Omega} A(x) \nabla u \cdot \nabla\left(G_{k}\left(|u|^{-p} u\right)\right) \mathrm{d} x \\
& =\int_{\Omega} g(x, u) G_{k}\left(|u|^{-p} u\right) \mathrm{d} x+\int_{\Omega} f G_{k}\left(|u|^{-p} u\right) \mathrm{d} x \\
& \leq \Lambda \int_{\Omega}|u|^{p-1}\left|G_{k}\left(|u|^{-p} u\right)\right| \mathrm{d} x+\int_{\Omega}|f|\left|G_{k}\left(|u|^{-p} u\right)\right| \mathrm{d} x .
\end{aligned}
$$

Therefore,

$$
(1-p) \lambda \int_{\left\{|u|^{1-p}>k\right\}}|u|^{-p}\left|\nabla u_{n}\right|^{2} \mathrm{~d} x \leq \Lambda|\Omega|+\int_{\Omega}|f||u|^{1-p} \mathrm{~d} x .
$$

Now we let $k$ goes to 0 on the left-hand side and by the monotone convergence Theorem,

$$
\lim _{k \rightarrow 0} \int_{\left\{|u|^{1-p}>k\right\}}|u|^{-p}|\nabla u|^{2}=\int_{\{|u|>0\}}|u|^{-p}|\nabla u|^{2}<+\infty .
$$

Applying Lemma 2.5 of [18], we obtain that $|u|^{1-(p / 2)} \in H_{0}^{1}(\Omega)$ and

$$
\int_{\{|u|>0\}}|u|^{-p}|\nabla u|^{2}=\int_{\Omega}|u|^{-p}|\nabla u|^{2}
$$

so that $|u|^{-p}|\nabla u|^{2} \in L^{1}(\Omega)$ as desired.

Remark 3.3. We point out that in case that the singular term satisfies

$$
g(x, s) s \geq 0 \quad \text { for all } s \in \mathbb{R}
$$


the minimum we have found is not trivial when $f \neq 0$. This is a consequence of comparing our minimum with the unique solution to the following problem

$$
\begin{cases}-\operatorname{div}(A(x) \nabla v)=f(x), & \text { in } \Omega \\ v=0, & \text { on } \partial \Omega,\end{cases}
$$

Observe that $f \neq 0$ implies $v \neq 0$. Moreover, it follows from condition (3.8) that $\mathcal{G}(x, s) \geq 0$ for all $s \in \mathbb{R}$, so that

$$
\mathcal{G}(x, v) \geq 0
$$

On the other hand, this function $v$ minimizes the functional defined on $H_{0}^{1}(\Omega)$ as

$$
\mathcal{J}_{0}[v]=\frac{1}{2} \int_{\Omega} A(x) \nabla v \cdot \nabla v \mathrm{~d} x-\int_{\Omega} f(x) v \mathrm{~d} x .
$$

Now appealing to the facts that $u$ minimizes $\mathcal{J}$ and $v$ minimizes $\mathcal{J}_{0}$ jointly with (3.9), we have the following inequalities

$$
\begin{aligned}
\mathcal{J}[u] \leq \mathcal{J}[v] & \leq \frac{1}{2} \int_{\Omega} A(x) \nabla v \cdot \nabla v \mathrm{~d} x-\int_{\Omega} \mathcal{G}(x, v) \mathrm{d} x-\int_{\Omega} f(x) v \mathrm{~d} x \\
& \leq \mathcal{J}_{0}[v]<\mathcal{J}_{0}[0]=0=\mathcal{J}[0] .
\end{aligned}
$$

This yields $u \neq 0$.

\section{Distributional solutions}

As pointed out a solution $u$ in the sense of Definition 2.1 need not be a solution in the sense of distributions to problem (1.1). Nevertheless, a sufficient condition which assure that $u$ is a solution in the sense of distributions is given below.

We begin with two natural hypotheses. To obtain a distributional solution, we require

$$
g(x, u) \in L_{l o c}^{1}(\Omega)
$$

Due to our singular setting, we also assume

$$
\lim _{s \rightarrow 0}|g(x, s)|=+\infty \quad \text { for almost all } x \in \Omega .
$$

A straightforward consequence of both assumptions is $|\{u=0\}|=0$, that we will use henceforth.

Lemma 4.1. Under the assumptions of Theorem 2.6 as well as (4.1) and (4.2), if $u$ is a solution to (1.1) in the sense of Definition 2.1, then the following equality holds,

$$
\begin{array}{rl}
\lim _{k \rightarrow 0} \frac{1}{k} \int_{\{|u|<k\}} \varphi A(x) \nabla u \cdot \nabla u & \mathrm{~d} x \\
=-\int_{\Omega} A(x) \nabla u \cdot \nabla \varphi \operatorname{sign} u \mathrm{~d} x+\int_{\Omega} \varphi g(x, u) \operatorname{sign} u \mathrm{~d} x+\int_{\Omega} \varphi f \operatorname{sign} u \mathrm{~d} x .
\end{array}
$$

for any $\varphi \in C_{0}^{\infty}(\Omega)$. 
Remark 4.2. We point out that, in the case that the stronger condition $g(x, u) \in L^{1}(\Omega)$ holds, the following proof actually applies to every $\varphi \in H^{1}(\Omega) \cap L^{\infty}(\Omega)$. Then, choosing $\varphi \equiv 1$, we get

$$
\lim _{k \rightarrow 0} \frac{1}{k} \int_{\{|u|<k\}} A(x) \nabla u \cdot \nabla u \mathrm{~d} x=\int_{\Omega} g(x, u) \operatorname{sign} u \mathrm{~d} x+\int_{\{u>0\}} f \mathrm{~d} x-\int_{\{u<0\}} f \mathrm{~d} x .
$$

Moreover, since $|\{u=0\}|=0, g(x, u)$ is defined a.e. in $\Omega$.

Proof. We first fix $k>0$, and choose $S(\tau)=\frac{1}{k} T_{k}(\tau)$ in (2.4). Then for any $\varphi \in C_{0}^{\infty}(\Omega)$, we have

$$
\begin{aligned}
\frac{1}{k} \int_{\{|u|<k\}} \varphi A(x) & \nabla u \cdot \nabla u \mathrm{~d} x+\frac{1}{k} \int_{\Omega} T_{k}(u) A(x) \nabla u \cdot \nabla \varphi \mathrm{d} x \\
= & \frac{1}{k} \int_{\Omega} g(x, u) T_{k}(u) \varphi \mathrm{d} x+\frac{1}{k} \int_{\Omega} f T_{k}(u) \varphi \mathrm{d} x .
\end{aligned}
$$

In order to let $k$ go to 0 , we evaluate each term in this equality. It is easy to verify that

$$
\lim _{k \rightarrow 0} \frac{1}{k} \int_{\Omega} T_{k}(u) A(x) \nabla u \cdot \nabla \varphi \mathrm{d} x=\int_{\Omega} A(x) \nabla u \cdot \nabla \varphi \operatorname{sign} u \mathrm{~d} x
$$

Moreover, since

$$
\frac{1}{k}\left|g(x, u) T_{k}(u)\right| \leq|g(x, u)|, \quad \text { a.e. in } \Omega .
$$

by Lebesgue's dominated convergence theorem we deduce

$$
\lim _{k \rightarrow 0} \frac{1}{k} \int_{\Omega} g(x, u) T_{k}(u) \varphi \mathrm{d} x=\int_{\{u \neq 0\}} g(x, u) \operatorname{sign} u \varphi \mathrm{d} x=\int_{\Omega} g(x, u) \operatorname{sign} u \varphi \mathrm{d} x .
$$

where we have used that $|\{u=0\}|=0$.

On the other hand, it follows

$$
\frac{1}{k} \int_{\Omega} \varphi f T_{k}(u) \mathrm{d} x=\frac{1}{k} \int_{\{u>0\}} \varphi f T_{k}(u) \mathrm{d} x+\frac{1}{k} \int_{\{u<0\}} \varphi f T_{k}(u) \mathrm{d} x,
$$

Letting $k$ go to zero, we conclude

$$
\lim _{k \rightarrow 0} \frac{1}{k} \int_{\Omega} \varphi f T_{k}(u) \mathrm{d} x=\int_{\{u>0\}} \varphi f \mathrm{~d} x-\int_{\{u<0\}} \varphi f \mathrm{~d} x=\int_{\Omega} \varphi f \operatorname{sign} u \mathrm{~d} x .
$$

Then (4.3) is a consequence of (4.5), (4.6), (4.7) and (4.8).

Theorem 4.3. Under the assumptions of Theorem 2.6 as well as (4.1) and (4.2), if $u \in H_{0}^{1}(\Omega)$ is a solution to (1.1) in the sense of Definition 2.1 and

$$
\lim _{k \rightarrow 0} \frac{1}{k} \int_{\{|u|<k\}} A(x) \nabla u \cdot \nabla u \mathrm{~d} x=0
$$

then $u$ is a distributional solution to problem (1.1). 
Remark 4.4. When $g(x, u) \in L^{1}(\Omega)$ holds, by previous Lemma and Remark 4.2, condition (4.9) is satisfied if the following equality holds true

$$
\int_{\{u>0\}} f \mathrm{~d} x+\int_{\{u>0\}} g(x, u) \mathrm{d} x=\int_{\{u<0\}} f \mathrm{~d} x+\int_{\{u<0\}} g(x, u) \mathrm{d} x .
$$

Proof. Fix $k>0$. We choose $S(s)=\frac{1}{k} T_{k}(|s|)$ in (2.4) obtaining

$$
\begin{aligned}
\frac{1}{k} \int_{\{|u|<k\}} \varphi \frac{u}{|u|} A(x) \nabla u \cdot \nabla u \mathrm{~d} x+\frac{1}{k} \int_{\Omega} T_{k}(|u|) A(x) \nabla u & \cdot \nabla \varphi \mathrm{d} x \\
& =\frac{1}{k} \int_{\Omega} \varphi g(x, u) T_{k}(|u|) \mathrm{d} x+\frac{1}{k} \int_{\Omega} \varphi f T_{k}(|u|) \mathrm{d} x,
\end{aligned}
$$

for any $\varphi \in C_{0}^{\infty}(\Omega)$.

Now we evaluate each term in this equality. Since

$$
\left|\int_{\{|u|<k\}} \varphi \frac{u}{|u|} A(x) \nabla u \cdot \nabla u \mathrm{~d} x\right| \leq\|\varphi\|_{\infty} C \int_{\{|u|<k\}}|\nabla u|^{2} \mathrm{~d} x,
$$

it follows that, by assumptions (4.9) and (1.2),

$$
\lim _{k \rightarrow 0} \frac{1}{k}\left|\int_{\{|u|<k\}} \varphi \frac{u}{|u|} A(x) \nabla u \cdot \nabla u \mathrm{~d} x\right| \leq\|\varphi\|_{\infty} C \lim _{k \rightarrow 0} \frac{1}{k} \int_{\{|u|<k\}}|\nabla u|^{2} \mathrm{~d} x=0 .
$$

On the other hand, it is straightforward that

$$
\lim _{k \rightarrow 0} \frac{1}{k} \int_{\Omega} T_{k}(|u|) A(x) \nabla u \cdot \nabla \varphi \mathrm{d} x=\int_{\{u \neq 0\}} A(x) \nabla u \cdot \nabla \varphi \mathrm{d} x=\int_{\Omega} A(x) \nabla u \cdot \nabla \varphi \mathrm{d} x,
$$

due to Stampacchia's theorem. Moreover, premise $|\{u=0\}|=0$ implies

$$
\lim _{k \rightarrow 0} \frac{1}{k} \int_{\Omega} \varphi g(x, u) T_{k}(|u|) \mathrm{d} x=\int_{\{u \neq 0\}} \varphi g(x, u) \mathrm{d} x=\int_{\Omega} \varphi g(x, u) \mathrm{d} x
$$

and

$$
\lim _{k \rightarrow 0} \frac{1}{k} \int_{\Omega} \varphi f T_{k}(|u|) \mathrm{d} x=\int_{\{u \neq 0\}} \varphi f \mathrm{~d} x=\int_{\Omega} \varphi f \mathrm{~d} x
$$

Combining (4.12), (4.13), (4.14) and (4.15), identity (4.11) becomes

$$
\int_{\Omega} A(x) \nabla u \cdot \nabla \varphi \mathrm{d} x=\int_{\Omega} \varphi g(x, u) \mathrm{d} x+\int_{\Omega} \varphi f \mathrm{~d} x,
$$

and the result is proved.

Remark 4.5. It is worth remarking that assumptions $g(x, s) \geq 0$ for all $s \in \mathbb{R}$ and $f \geq 0$ lead to a positive solution. Indeed, this fact follows from the inequality

$$
-\operatorname{div}(A(x) \nabla u)=g(x, u)+f(x) \geq f(x)
$$


by applying the strong maximum principle. Then, for every $\omega \subset \subset \Omega$ there exists a constant $C_{\omega}>0$ such that $u \geq C_{\omega}$ a.e. in $\omega$. As a consequence, $g(x, u) \in L_{l o c}^{1}(\Omega)$ and $g(x, u) \varphi \in L^{1}(\Omega)$ for all test functions $\varphi$ with compact support and so $u$ is a solution in the sense of distributions. Furthermore, we may also deduce that $g(x, u) \varphi \in L^{1}(\Omega)$ for all $\varphi \in H_{0}^{1}(\Omega)$. Nevertheless, identity (4.10) does not hold, since its right hand side vanishes. Therefore, $\varphi \equiv 1$ cannot be chosen as a test function, so that $g(x, u) \notin L^{1}(\Omega)$.

\section{Uniqueness of POSITIVE SOlutions: PROOF OF TheOREM 2.7}

Let $u_{1}, u_{2}$ be two nonnegative solutions to problem (1.1) which satisfy condition (2.6) with $u$ replaced by $u_{i}$, $i=1,2$. Moreover, to get a contradiction, assume that

$$
\left|\left\{x \in \Omega: u_{1}(x)>u_{2}(x)\right\}\right|>0 .
$$

Since $u_{1}$ is a solution to (1.1), we choose $S(t)=h_{\delta}(t)$ in Definition 2.1 written for $u=u_{1}$, where

$$
h_{\delta}(t)= \begin{cases}0, & |t| \leq \delta \\ \frac{t}{\delta} \operatorname{sign} t-1 & \delta \leq|t| \leq 2 \delta \\ 1 & |t| \geq 2 \delta,\end{cases}
$$

for any $\delta>0$. The following equality holds true

$$
\begin{gathered}
\int_{\Omega} A(x) \nabla u_{1} \cdot \nabla u_{1} h_{\delta}^{\prime}\left(u_{1}\right) \varphi \mathrm{d} x+\int_{\Omega} h_{\delta}\left(u_{1}\right) A(x) \nabla u_{1} \cdot \nabla \varphi \mathrm{d} x \\
=\int_{\Omega} g\left(x, u_{1}\right) h_{\delta}\left(u_{1}\right) \varphi \mathrm{d} x+\int_{\Omega} f h_{\delta}\left(u_{1}\right) \varphi \mathrm{d} x,
\end{gathered}
$$

for every $\varphi \in H^{1}(\Omega) \cap L^{\infty}(\Omega)$.

Now let us choose $\varphi=G_{m}\left(\left(u_{1}-u_{2}\right)_{+}\right), m>2 \delta$, in the previous equality. We have

$$
\begin{aligned}
& \int_{\Omega} A(x) \nabla u_{1} \cdot \nabla u_{1} h_{\delta}^{\prime}\left(u_{1}\right) G_{m}\left(\left(u_{1}-u_{2}\right)_{+}\right) \mathrm{d} x \\
& \quad+\int_{\Omega} h_{\delta}\left(u_{1}\right) A(x) \nabla u_{1} \cdot \nabla\left(u_{1}-u_{2}\right) G_{m}^{\prime}\left(\left(u_{1}-u_{2}\right)_{+}\right) \mathrm{d} x \\
& \quad=\int_{\Omega} g\left(x, u_{1}\right) h_{\delta}\left(u_{1}\right) G_{m}\left(\left(u_{1}-u_{2}\right)_{+}\right) \mathrm{d} x+\int_{\Omega} f h_{\delta}\left(u_{1}\right) G_{m}\left(\left(u_{1}-u_{2}\right)_{+}\right) \mathrm{d} x .
\end{aligned}
$$

Since $u_{2}$ is a solution to (1.1), we can choose $S(t)=h_{\delta}(t)$ in Definition 2.1 written for $u=u_{2}$ and $\varphi=$ $G_{m}\left(\left(u_{1}-u_{2}\right)_{+}\right)$. We obtain an analogous equality, i.e..

$$
\begin{aligned}
\int_{\Omega} & A(x) \nabla u_{2} \cdot \nabla u_{2} h_{\delta}^{\prime}\left(u_{2}\right) G_{m}\left(\left(u_{1}-u_{2}\right)_{+}\right) \mathrm{d} x \\
& +\int_{\Omega} h_{\delta}\left(u_{2}\right) A(x) \nabla u_{2} \cdot \nabla\left(u_{1}-u_{2}\right) G_{m}^{\prime}\left(\left(u_{1}-u_{2}\right)_{+}\right) \mathrm{d} x \\
\quad & \left.=\int_{\Omega} g\left(x, u_{2}\right) h_{\delta}\left(u_{2}\right)\right) G_{m}\left(\left(u_{1}-u_{2}\right)_{+}\right) \mathrm{d} x+\int_{\Omega} f h_{\delta}\left(u_{2}\right) G_{m}\left(\left(u_{1}-u_{2}\right)_{+}\right) \mathrm{d} x
\end{aligned}
$$


Subtracting these equalities, we get

$$
\begin{aligned}
& \int_{\Omega} h_{\delta}\left(u_{1}\right) A(x) \nabla u_{1} \cdot \nabla\left(u_{1}-u_{2}\right) G_{m}^{\prime}\left(\left(u_{1}-u_{2}\right)_{+}\right) \mathrm{d} x \\
- & \int_{\Omega} h_{\delta}\left(u_{2}\right) A(x) \nabla u_{2} \cdot \nabla\left(u_{1}-u_{2}\right) G_{m}^{\prime}\left(\left(u_{1}-u_{2}\right)_{+}\right) \mathrm{d} x \\
& +\frac{1}{\delta} \int_{\left\{\delta \leq\left|u_{1}\right| \leq 2 \delta\right\}} A(x) \nabla u_{1} \cdot \nabla u_{1} G_{m}\left(\left(u_{1}-u_{2}\right)_{+}\right), \mathrm{d} x \\
& -\frac{1}{\delta} \int_{\left\{\delta \leq\left|u_{2}\right| \leq 2 \delta\right\}} A(x) \nabla u_{2} \cdot \nabla u_{2} G_{m}\left(\left(u_{1}-u_{2}\right)_{+}\right) \mathrm{d} x \\
= & \int_{\left\{u_{1}-u_{2}>m\right\}}\left[g\left(x, u_{1}\right)+f\right] h_{\delta}\left(u_{1}\right) G_{m}\left(\left(u_{1}-u_{2}\right)_{+}\right) \mathrm{d} x \\
- & \int_{\left\{u_{1}-u_{2}>m\right\}}\left[g\left(x, u_{2}\right)+f\right] h_{\delta}\left(u_{2}\right) G_{m}\left(\left(u_{1}-u_{2}\right)_{+}\right) \mathrm{d} x
\end{aligned}
$$

Now we pass to the limit for $\delta$ which goes to 0 in the last equality.

Since $h_{\delta}\left(u_{i}\right) \rightarrow 1$ in $\left\{u_{i}>0\right\}$ and $\nabla u_{i}=0$ in $\left\{u_{i}=0\right\}$, by Lebesgue's dominated convergence theorem, we get

$$
\begin{gathered}
\lim _{\delta \rightarrow 0}\left(\int_{\Omega} h_{\delta}\left(u_{1}\right) A(x) \nabla u_{1} \nabla\left(u_{1}-u_{2}\right) G_{m}^{\prime}\left(\left(u_{1}-u_{2}\right)_{+}\right) \mathrm{d} x\right. \\
\left.-\int_{\Omega} h_{\delta}\left(u_{2}\right) A(x) \nabla u_{2} \nabla\left(u_{1}-u_{2}\right) G_{m}^{\prime}\left(\left(u_{1}-u_{2}\right)_{+}\right) \mathrm{d} x\right) \\
=\int_{\Omega} A(x) \nabla G_{m}\left(\left(u_{1}-u_{2}\right)_{+}\right) \cdot \nabla G_{m}\left(\left(u_{1}-u_{2}\right)_{+}\right) \mathrm{d} x .
\end{gathered}
$$

By assumption (2.6), we have

$$
\begin{aligned}
& \lim _{\delta \rightarrow 0} \frac{1}{\delta} \int_{\left\{\delta \leq\left|u_{1}\right| \leq 2 \delta\right\}} A(x) \nabla u_{1} \cdot \nabla u_{1} G_{m}\left(\left(u_{1}-u_{2}\right)_{+}\right), \mathrm{d} x=0 \\
& \lim _{\delta \rightarrow 0} \frac{1}{\delta} \int_{\left\{\delta \leq\left|u_{2}\right| \leq 2 \delta\right\}} A(x) \nabla u_{2} \cdot \nabla u_{2} G_{m}\left(\left(u_{1}-u_{2}\right)_{+}\right) \mathrm{d} x=0
\end{aligned}
$$

Denote

$$
\begin{aligned}
I_{1, \delta} & =\int_{\left\{u_{1}-u_{2}>m\right\}}\left[g\left(x, u_{1}\right)+f\right] h_{\delta}\left(u_{1}\right) G_{m}\left(\left(u_{1}-u_{2}\right)_{+}\right) \mathrm{d} x, \\
I_{2, \delta} & =\int_{\left\{u_{1}-u_{2}>m\right\}}\left[g\left(x, u_{2}\right)+f\right] h_{\delta}\left(u_{2}\right) G_{m}\left(\left(u_{1}-u_{2}\right)_{+}\right) \mathrm{d} x, .
\end{aligned}
$$

Let us evaluate the limit as $\delta$ goes to 0 of the right-hand side in (5.1):

$$
\limsup _{\delta \rightarrow 0}\left(I_{1, \delta}-I_{2, \delta}\right)=\limsup _{\delta \rightarrow 0} I_{1, \delta}-\liminf _{\delta \rightarrow 0} I_{2, \delta}
$$


By Lebesgue theorem we have

$$
\lim _{\delta \rightarrow 0} I_{1, \delta}=\int_{\left\{u_{1}-u_{2}>m\right\}}\left[g\left(x, u_{1}\right)+f\right]\left(G_{m}\left(u_{1}-u_{2}\right)\right)_{+} \mathrm{d} x=A .
$$

Indeed, since $u_{i}$ are nonnegative functions,

$$
\left[g\left(x, u_{1}\right)+f\right] \leq \frac{1}{u_{1}^{1-p}}+f \leq \frac{1}{\left(u_{2}+m\right)^{1-p}}+f \leq \frac{1}{(m)^{1-p}}+f .
$$

On the other hand, by Fatou's lemma

$$
-\liminf _{\delta \rightarrow 0} I_{2, \delta} \leq-\int_{\left\{u_{1}-u_{2}>m\right\}}\left[g\left(x, u_{2}\right)+f\right]\left(G_{m}\left(u_{1}-u_{2}\right)\right)_{+} \mathrm{d} x=-B
$$

Assume that $B<+\infty$. Since $g(x, \cdot)$ is a nonincreasing function, combining (5.5), (5.6) and (5.7), we obtain

$$
\limsup _{\delta \rightarrow 0}\left(I_{1, \delta}-I_{2, \delta}\right)=A-B \leq 0
$$

Collecting (5.1)-(5.4), (5.8), by ellipticity condition (1.2), we deduce

$$
\int_{\Omega}\left|\nabla G_{m}\left(\left(u_{1}-u_{2}\right)+\right)\right|^{2} \mathrm{~d} x=0
$$

that is a contradiction. If $B=+\infty$, in analogous way we arrive at

$$
\limsup _{\delta \rightarrow 0}\left(I_{1, \delta}-I_{2, \delta}\right)=-\infty
$$

which yields again a contradiction. We conclude that $u_{1} \leq u_{2}$ a.e. in $\Omega$. In the same way we can prove $u_{2} \leq u_{1}$ a.e. in $\Omega$ and the conclusion follows.

\section{Remarks on EXISTENCE USING AN APPROXIMATION APPROACH}

This section is devoted to some remarks concerning the standard approximation approach to existence for problem (1.1). Such an approach consists in considering suitable approximating problems and to get a priori estimates for solutions to these problems and their gradients. These a priori estimates allow to find a function $u$ which is the limit of a subsequence of approximate solutions and which should become the solution of our problem.

A sequence of approximate problems is given by

$$
\begin{cases}-\operatorname{div}\left(A(x) \nabla u_{n}\right)=g_{n}\left(x, u_{n}\right)+f(x), & \text { in } \Omega \\ u_{n}=0 & \text { on } \partial \Omega\end{cases}
$$

for certain non singular functions $g_{n}$ which may be defined in different ways. For instance:

$$
g_{n}(x, s)= \begin{cases}\frac{1+t}{2} g\left(x, \frac{1}{n}\right)+\frac{1-t}{2} g\left(x, \frac{-1}{n}\right) & \text { if } s=\frac{t}{n} \text { with }|t| \leq 1 \\ g(x, s) & \text { if }|s| \geq \frac{1}{n} .\end{cases}
$$


In case $g\left(x, \frac{1}{n}\right)$ and $g\left(x, \frac{-1}{n}\right)$ are both positive or both negative, we may consider problem (6.1) with $g_{n}(x, s)=$ $T_{n}(g(x, s))$. Notice, however, that $g\left(x, \frac{1}{n}\right)$ and $g\left(x, \frac{-1}{n}\right)$ may have different signs.

For any fixed $n \in \mathbb{N}$, equation (6.1) is the Euler-Lagrange equation of the approximate functional (see, for instance, [14], Chap. 8)

$$
\mathcal{J}_{n}[u]=\frac{1}{2} \int_{\Omega} A(x) \nabla u \cdot \nabla u-\int_{\Omega} \mathcal{G}_{n}(x, u)-\int_{\Omega} f(x) u,
$$

where $\mathcal{G}_{n}(x, s)$ denotes the primitive of $g_{n}(x, s)$, i.e..

$$
\mathcal{G}_{n}(x, s)=\int_{0}^{s} g_{n}(x, t) \mathrm{d} t .
$$

It is easy to verify a uniform bound

$$
\left|\mathcal{G}_{n}(x, s)\right| \leq \Lambda \frac{|s|^{p}}{p}
$$

As proved for $\mathcal{J}$, we can see that a minimizer $u_{n} \in H_{0}^{1}(\Omega) \cap L^{\infty}(\Omega)$ of $\mathcal{J}_{n}$, for any fixed $n \in \mathbb{N}$, exists and therefore $u_{n}$ is a weak solution to this problem (6.1).

By means of usual techniques, we can check that the sequence of approximate solutions $\left(u_{n}\right)_{n}$ satisfies a priori estimates in $L^{\infty}(\Omega)$ and in $H_{0}^{1}(\Omega)$. By these estimates we deduce that $u_{n}$ converges to a function $u \in H_{0}^{1}(\Omega)$ which should be the sought solution.

It is not difficult to prove that $u$ minimizes functional $\mathcal{J}$, while some difficulties appear in proving that $u$ satisfies (2.4). Indeed in order to directly check that condition (2.4) holds, we need to have the strong convergence in $L^{2}\left(\Omega ; \mathbb{R}^{N}\right)$

$$
\nabla G_{k}\left(u_{n}\right) \rightarrow \nabla G_{k}(u) \quad \text { for all } k>0
$$

but we are not able to obtain it when functions $u_{n}$ change sign. As an alternative, we must apply Proposition 3.2 to see that assertion (2.4) holds. Hence, this approach requires going through the minimization of the functional and does not improve our proof of Theorem 2.6.

This approach, however, allows us to get another sufficient condition to guarantee that $u$ is a solution in the sense of distributions. To see this, we take $v \in H_{0}^{1}(\Omega)$ as test function in (6.1) and so

$$
\int_{\Omega} A(x) \nabla u_{n} \cdot \nabla v \mathrm{~d} x=\int_{\Omega} g_{n}\left(x, u_{n}\right) v \mathrm{~d} x+\int_{\Omega} f v \mathrm{~d} x .
$$

After applying the Hölder and Sobolev inequalities and using the a priori estimates in $H_{0}^{1}(\Omega)$, it yields

$$
\begin{aligned}
\left|\int_{\Omega} g_{n}\left(x, u_{n}\right) v \mathrm{~d} x\right| \leq \int_{\Omega} \mid A(x) \nabla u_{n} & \nabla v\left|\mathrm{~d} x+\int_{\Omega}\right| f|| v \mid \mathrm{d} x \\
& \leq C\left\|\nabla u_{n}\right\|_{2}\|\nabla v\|_{2}+\|f\|_{\frac{2 N}{N+2}}\|v\|_{2^{*}} \\
& \leq C\left[\left\|\nabla u_{n}\right\|_{2}+\|f\|_{\frac{2 N}{N+2}}\right]\|\nabla v\|_{2}<+\infty
\end{aligned}
$$

So, one deduces that the sequence $\left(g_{n}\left(x, u_{n}\right)\right)_{n}$ is bounded in the dual space $H^{-1}(\Omega)$. Hence, up to subsequences, we can find $\xi \in H^{-1}(\Omega)$ such that

$$
g_{n}\left(x, u_{n}\right) \rightarrow \xi
$$


As a consequence, letting $n \rightarrow \infty$ in (6.4), we obtain

$$
\int_{\Omega} A(x) \nabla u \cdot \nabla v \mathrm{~d} x=\langle\xi, v\rangle_{H^{-1}, H_{0}^{1}}+\int_{\Omega} f v \mathrm{~d} x
$$

for all $v \in H_{0}^{1}(\Omega)$. It suggests that $\xi$ plays the role of $g(x, u)$ in some sense. Therefore, the identity $\xi=g(x, u) \in$ $L_{l o c}^{1}(\Omega)$ implies that $u$ is solution in the sense of distributions.

Actually, we just know that

$$
\langle\xi, S(u) \varphi\rangle_{H^{-1}, H_{0}^{1}}=\int_{\Omega} g(x, u) S(u) \varphi \mathrm{d} x
$$

for every Lipschitz-continuous function $S$ satisfying $S(0)=0$ and every $\varphi \in H^{1}(\Omega)$.

Example 6.1. The case $g(x, s)=-|s|^{p-2} s$ is illustrative of the difficulties of addressing these singular problems. In this example we choose

$$
g_{n}(x, s)= \begin{cases}-\frac{1+t}{2} n^{1-p}+\frac{1-t}{2} n^{1-p} & \text { if } s=\frac{t}{n} \text { with }|t| \leq 1 \\ -|s|^{p-2} s & \text { if }|s| \geq \frac{1}{n} .\end{cases}
$$

Observe that $g_{n}(x, s) s \leq 0$ for all $n \in \mathbb{N}$. Taking $T_{k}\left(u_{n}\right)$ as test function, we get

$$
\int_{\Omega}\left|\nabla T_{k}\left(u_{n}\right)\right|^{2} d x-\int_{\Omega} g_{n}\left(x, u_{n}\right) T_{k}\left(u_{n}\right) \mathrm{d} x=\int_{\Omega} f T_{k}\left(u_{n}\right) \mathrm{d} x
$$

and disregarding a nonnegative term, we deduce

$$
\frac{1}{k} \int_{\Omega}\left|g_{n}\left(x, u_{n}\right)\right|\left|T_{k}\left(u_{n}\right)\right| \mathrm{d} x \leq \int_{\Omega}|f| \mathrm{d} x
$$

Thus, Fatou's Lemma yields

$$
\frac{1}{k} \int_{\Omega}|g(x, u)|\left|T_{k}(u)\right| \mathrm{d} x \leq \int_{\Omega}|f| \mathrm{d} x
$$

and, letting now $k$ go to $\infty$, it leads to

$$
\int_{\{u \neq 0\}}|g(x, u)| \mathrm{d} x \leq \int_{\Omega}|f| \mathrm{d} x
$$

wherewith $|u|^{p-2} u \chi_{\{u \neq 0\}} \in L^{1}(\Omega)$.

Observe that we cannot deduce $|u|^{p-2} u \in L^{1}(\Omega)$. Indeed, if $f=0$, then $u=0$. Moreover, the element $\xi$ occurring in (6.5) satisfies $\xi=0$. Obviously, $|\{u=0\}| \neq 0$, so that $\xi \neq|u|^{p-2} u$ and $u$ is not a solution in the sense of distributions.

Acknowledgements. The authors are grateful to David Arcoya for useful discussions and suggestions. They also thank Referees for carefully reading our manuscript and giving constructive comments which improved this paper. Finally the authors would like to thank University of Campania "L. Vanvitelli", University of Naples Federico II and University of Valencia for supporting some visiting appointments and their kind hospitality. 


\section{REFERENCES}

[1] D. Arcoya and L. Boccardo, Multiplicity of solutions for a Dirichlet problem with a singular and a supercritical nonlinearities. Differ. Integr. Equ. 26 (2013) 119-128.

[2] D. Arcoya and L. Moreno-Mérida, Multiplicity of solutions for a Dirichlet problem with a strongly singular nonlinearity. Nonlinear Anal. 95 (2014) 281-291.

[3] L. Boccardo, A Dirichlet problem with singular and supercritical nonlinearities. Nonlinear Anal. 75 (2012) $4436-4440$.

[4] L. Boccardo and J. Casado-Díaz, Some properties of solutions of some semilinear elliptic singular problems and applications to the G-convergence. Asymptot. Anal. 86 (2014) 1-15.

[5] L. Boccardo and L. Orsina, Semilinear elliptic equations with singular nonlinearities. Calc. Var. Partial Differ. Equ. 37 (2010) 363-380.

[6] J. Casado-Díaz and F. Murat, Semilinear problems with right-hand sides singular at $u=0$ which change sig. To appear in Ann. Inst. Henri Poincaré, Anal. Non Linéaire. doi:10.1016/j.anihpc.2020.09.00

[7] A. Canino and M. Degiovanni, A variational approach to a class of singular semilinear elliptic equations. J. Convex Anal. 11 (2004) $147-162$.

[8] A. Canino, M. Grandinetti and B. Sciunzi, Symmetry of solutions of some semilinear elliptic equations with singular nonlinearities. J. Differ. Equ. 255 (2013) 4437-4447.

[9] A. Canino, F. Esposito and B. Sciunzi, On the Höpf boundary lemma for singular semilinear elliptic equations. J. Differ. Equ. 266 (2019) 5488-5499.

[10] B. Brandolini, F. Chiacchio and C. Trombetti, Symmetrization for singular semilinear elliptic equations. Ann. Mat. Pura Appl. 193 (2014) 389-404.

[11] M.G. Crandall, P.H. Rabinowitz and L. Tartar, On a Dirichlet problem with a singular nonlinearity. Commun. Partial Differ. Equ. 2 (1977) 193-222.

[12] G. Croce, An elliptic problem with two singularities. Asymptot. Anal. 78 (2012) 1-10.

[13] G. Dal Maso, F. Murat, L. Orsina and A. Prignet, Renormalized solutions of elliptic equations with general measure data. Ann. Scuola Norm. Sup. Pisa Cl. Sci. 28 (1999) 741-808.

[14] L.C. Evans, Partial differential equations. Vol. 19 of Graduate Studies in Mathematics. American Mathematical Society, Providence, RI (1998).

[15] D. Giachetti, P.J. Martínez-Aparicio and F. Murat, On the definition of the solution to a semilinear elliptic problem with a strong singularity at $u=0$. Nonlinear Anal. 177 (2018) 491-523.

[16] D. Giachetti, P.J. Martínez-Aparicio and F. Murat, Definition, existence, stability and uniqueness of the solution to a semilinear elliptic problem with a strong singularity at $u=0$. Ann. Sc. Norm. Super. Pisa Cl. Sci. 18 (2018) 1395-1442.

[17] D. Giachetti, P.J. Martínez-Aparicio and F. Murat, A semilinear elliptic equation with a mild singularity at $u=0:$ existence and homogenization. J. Math. Pures Appl. 107 (2017) 41-77.

[18] D. Giachetti, F. Petitta and S. Segura de León, Elliptic equations having a singular quadratic gradient term and a changing sign datum. Comm. Pure Appl. Anal. 11 (2012) 1875-1895.

[19] E. Giusti, Direct methods in the calculus of variations. World Scientific, Singapore (2003).

[20] P.-L. Lions and F. Murat, Sur les solutions renormalisées d'équations elliptiques non linéaires. Unpublished paper.

[21] F. Oliva and F. Petitta, On singular elliptic equations with measure sources. ESAIM: COCV 22 (2016) $289-308$.

[22] L. Orsina and F. Petitta, A Lazer-McKenna type problem with measures. Differ. Integr. Equ. 29 (2016) 19-36. 